\title{
Differentiation of bland from neoplastic thrombus of the portal vein in patients with hepatocellular carcinoma: application of susceptibility-weighted MR imaging
}

Chuanming $\mathrm{Li}^{1}$, Jiani Hư ${ }^{2}$ Daiquan Zhou' ${ }^{1}$ Jun Zhao ${ }^{1}$, Kuansheng $\mathrm{Ma}^{3}$, Xuntao Yin ${ }^{{ }^{*}}$ and Jian Wang ${ }^{1 *}$

\begin{abstract}
Background: Neoplastic and bland portal vein thrombi (PVT) are both common in patients with hepatocellular carcinoma (HCC). The correct discrimination of them is essential for therapeutic strategies planning and survival predicting. The current study aims to investigate the value of susceptibility-weighted imaging (SWI) in differentiating bland from neoplastic PVT in HCC patients.

Methods: 20 HCC patients with bland PVT and 22 HCC patients with neoplastic PVT were imaged with non-contrast SWI at 3.0 Tesla MRI. The signal intensity (SI) of the PVT and HCC lesions in the same patients was compared on SW images. The phase values of the PVT were compared between neoplastic and bland thrombi cohorts. Receiver operator characteristics (ROC) analysis was conducted to evaluate the diagnostic ability of the phase values for neoplastic and bland thrombi discrimination.

Results: 20 of 22 neoplastic PVT were judged similar SI and 2 were judged lower SI than their HCC. For 20 bland PVT, 19 were judged lower SI and 1 was judged similar SI as their HCC $(P<0.001)$. The average phase values $(0.361 \pm 0.224)$ of the bland PVT were significantly higher than those of the neoplastic PVT $(-0.328 \pm 0.127, P<0.001)$. The AUC for phase values in differentiating bland from neoplastic PVT was 0.989. The best cut-off value was -0.195 , which gave a sensitivity of $95 \%$ and a specificity of $95.5 \%$.
\end{abstract}

Conclusions: SW imaging appears to be a promising new method for distinguishing neoplastic from bland PVT. The high sensitivity and specificity suggest its high value in clinical practice.

Keywords: MRI, Susceptibility-weighted imaging, Thrombosis, Portal vein, Hepatocellular carcinoma

\section{Background}

Portal vein thrombosis is a form of venous thrombosis affecting the hepatic portal vein, which can lead to portal hypertension and a reduction in the blood supply to the liver. Neoplastic portal vein thrombus is found in $6.5 \%-44 \%$ of patients with hepatocellular carcinoma (HCC). It renders a patient unsuitable for aggressive treatment approaches, such as surgical resection or chemoembolization, due to the unusually high incidence of tumor recurrence [1-3]. Bland thrombus occurs in

\footnotetext{
*Correspondence: willyxt@163.com; wangjian_1964@sohu.com 'Department of Radiology, Southwest Hospital, Third Military Medical University, 30 Gaotanyan Road, Chongqing 400038, China Full list of author information is available at the end of the article
}

$4.5 \%-26 \%$ of patients with chronic liver disease and in $42 \%$ of patients with HCC. It can be resolved after thrombolytic and anticoagulant therapy $[4,5]$. Neoplastic and bland portal vein thrombi discrimination is of great clinical significance for determining the therapeutic approach, predicting survival, and assessing candidates for liver transplantation.

$\mathrm{T} 2^{*}$-weighted imaging (T2*WI) is sensitive to ferrihemoglobin and hemosiderin based on the local field inhomogeneity generated by the paramagnetic effect of iron particles. T2* WI has been proven useful in cerebral venous thrombosis detection and evaluation [6]. Susceptibility-weighted imaging (SWI), which exploits the susceptibility differences between tissues as a new 
type of contrast, is more sensitive to detecting focal field inhomogeneity by adding phase information to the T2* contrast. SWI phase imaging avoids the impact of main magnetic field inhomogeneity through the implementation of a high-pass filter [7]. Three-dimensional (3D) SWI has been proven superior to $\mathrm{T} 2 *$ and other existing magnetic resonance imaging (MRI) techniques for the detection of iron content and hemorrhage in brain $[8,9]$. Two-dimensional SWI is a newer approach than 3D SWI, and it is nearly immune to breathing artifacts because it takes advantage of breath-holds. This technique has been successfully applied to the analysis of cirrhotic livers [10]. The value of SW imaging has not, to our knowledge, been studied for characterizing intravascular thrombosis in the liver. The purpose of this study was to investigate the value of SW imaging in distinguishing a bland thrombus from a neoplastic thrombus of the portal vein in patients with HCC.

\section{Methods}

\section{Subjects}

This HIPAA-compliant study was approved by the ethics commission of Southwest Hospital of China and written informed consent was obtained from each patient. From Oct 2011 to Dec 2013, 46 consecutive patients who had pathology-confirmed HCC and portal vein thrombus (PVT) participated in this study. 4 patients were excluded because of the following: a history of hepatic surgery, coexisting bland and neoplastic thrombi, or an unsuccessful examination resulting from body movement and artifacts. Thus, a total of 42 patients (20 men and 22 women, with a mean age of 45.3 years, range of 36-65 years; a mean weight of $71.56 \mathrm{~kg}$, range of $47-98 \mathrm{~kg}$ ) including 22 neoplastic PVT and 20 bland PVT formed the final study cohort. The PVT was localized in: the main portal trunk in 12 cases, the right branch in 16 cases, the left branch in 10 cases, and a combination of these in 4 cases. PVT of 22 patients were confirmed by surgery, 10 were confirmed by biopsy, and 10 were diagnosed based on other imaging criteria according to the litarature [11-14].

\section{Computed tomographic imaging}

Contrast-enhanced multiphase CT was performed with a multi-detector dual-source CT (Definition, Siemens Healthcare, Forchheim, Germany). The examination consisted of precontrast images and three dynamic phase images acquired $35 \mathrm{~s}$ (hepatic arterial phase), $70 \mathrm{~s}$ (portal venous phase), and $180 \mathrm{~s}$ (delayed phase) following the intravenous administration of 100-120 ml Ultravist 370 (Bayer-Schering, Leverkusen, Germany) at a rate of $3-4 \mathrm{ml} / \mathrm{s}$. The imaging parameters were as follows: $250 \mathrm{mAs}, 120 \mathrm{kVp}$, and $1.2 \mathrm{~mm}$ beam collimation with a $0.5 \mathrm{~s}$ gantry rotation time. The field of view (FOV) was $35 \mathrm{~cm}$, with a reconstruction thickness and interval of $5 \mathrm{~mm}$.

\section{Magnetic resonance imaging}

MR imaging was performed with a $3.0 \mathrm{~T}$ whole body system (Magnetom Trio, Siemens Healthcare, Erlangen, Germany) using a standard 12-channel matrix coil without intravenous contrast enhancement. The following MR pulse sequences were used: transverse T1-weighted 2D gradient echo (GRE) (flip angle $70^{\circ}$, TR/TE 140/2.46 ms), transverse T2-weighted 2D fast spin echo (flip angle $122^{\circ}$, $\mathrm{TR} / \mathrm{TE}=3700 / 84 \mathrm{~ms}$ ) and transverse abdominal 2D SWI (flip angle $20^{\circ}, \mathrm{TR} / \mathrm{TE}=150 / 10 \mathrm{~ms}$ ). For all of the patients, the following parameters were used: FOV $280 \times 285 \mathrm{~mm}^{2}$; matrix $384 \times 250$; 30 slices; and a slice thickness of $5 \mathrm{~mm}$ with a gap of $1 \mathrm{~mm}$. The protocol for SWI was similar to that used in a previous study [10]. Three breath-holds were used, each lasting 16 seconds. The total acquisition time was not longer than 1 minute and 20 seconds, including the break time between the breath holds. SWI postprocessing was done inline and consisted of the following steps: 1) Original images from each channel were passed through a $32 \times 32$ high pass filter to remove background artifacts; 2) The highpass filtered images from each channel were weighted by the coil sensitivity factor and combined to generate a single complex image; 3) highpass filter corrected phase images were created from the final complex images; 4) a normalized phase mask was calculated from each corrected phased image and multiplied with the magnitude image to produce the final SWI and phase image [7].

\section{Image analysis}

All of the SWI images were evaluated with SPIN software (Signal Processing in NMR, Version 1751, MRI Institute for Biomedical Research, Detroit, MI, USA; http://www.mrimaging.com/category.88.html) by two reviewers who were unaware of the bland or neoplastic nature of the thrombi and who had no access to the other sequences. All SW Images were evaluated qualitatively and then quantitatively.

\section{Qualitative analysis}

For qualitative analysis, the readers were asked to compare the signal intensity (SI) of the portal vein thrombi with those of the HCC on the SW images. They classified the SI of the portal vein thrombi into the following categories: higher, equal to, or lower than the SI of the HCC. The readings were performed separately.

\section{Quantitative analysis}

Regions of interest (ROI) were drawn directly to delineate the entire HCC and PVT, avoiding any vessels and hemorrhages (Figure 1). The mean and standard deviation (SD) of the Siemens Phase Unit (SPU) were obtained from the 


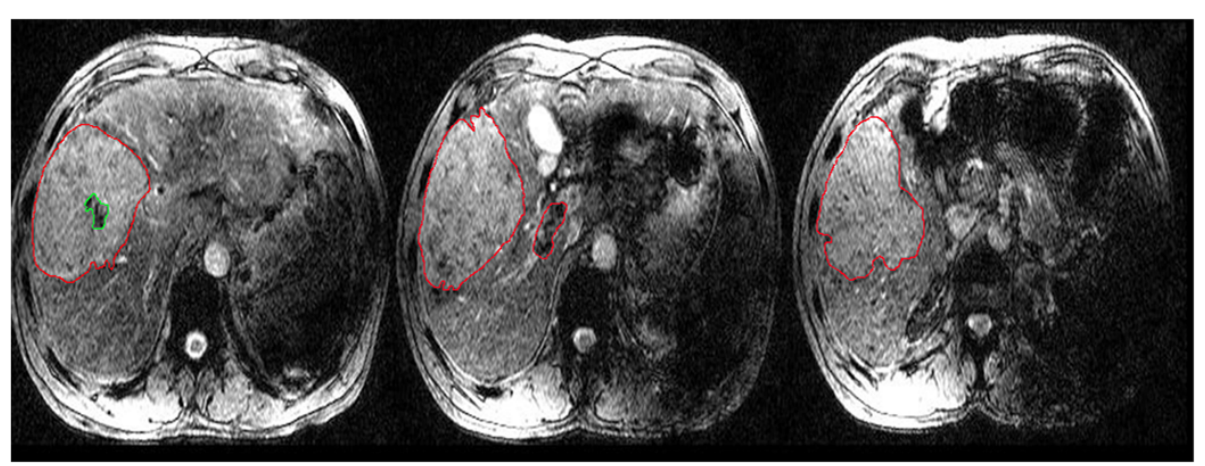

Figure 1 Example of the ROI over the tumor and thrombus. Red lines delineate the HCC and PVT, green lines delineate a hemorrhages in the HCC.

entire ROIs and converted into radians using the following equation: (SPU-2048) $x \pi / 2048$ [15]. The phase values of the tumors in the neoplastic and bland cohorts were compared using the Mann-Whitney test. The phase values of the tumors and thrombi were compared in each group using the Wilcoxon matched pairs signed rank test. The phase values of the thrombi of the two cohorts were compared using the Mann-Whitney test. P values $<0.05$ were considered statistically significant. Receiver operator characteristics (ROC) analysis was conducted to evaluate the diagnostic ability of phase values for neoplastic and bland thrombi discrimination. The areas under the ROC curve (AUC) and the confidence intervals (CIs) were assessed. The cut-off values that maximized the sum of the sensitivity and specificity were determined and set as the point in the most upper left hand corner. All statistical analyses were performed with the SPSS 17.0 software package (SPSS Inc., Chicago, IL, USA).

\section{Results}

Qualitative analysis

20 of 22 neoplastic thrombi were judged similar SI and 2 were judged lower SI than their HCC. For 20 bland thrombi, 19 were judged lower SI and 1 were judged similar $\mathrm{SI}$ as their HCC $(P<0.001)$ (Figures 2 and 3$)$. There is no significant difference between the two readers $(P>0.05)$.

\section{Quantitative analysis}

There is a significant phase value difference between bland thrombi $(0.361 \pm 0.224)$ and neoplastic thrombi $(-0.328 \pm 0.127, P<0.001)$. The AUC for phase values in differentiating bland from neoplastic PVT was 0.989. The best cut-off value was -0.195 , which gave a sensitivity of 95\% and a specificity of $95.5 \%$ (Figures 4 and 5 ).

No statistically significant difference was found between the phase values of the HCC in the neoplastic and bland cohorts $(-0.340 \pm 0.067$, and $-0.326 \pm 0.049$ respectively; $P>0.05)$. No statistically significant difference was found between the phase values of the thrombi $(-0.328 \pm 0.127)$ and the HCC $(-0.340 \pm 0.067)$ in the neoplastic PVT group $(P>0.05)$. The phase values of the thrombi $(0.361 \pm 0.224)$ were significantly higher than those of the corresponding HCC $(-0.326 \pm 0.049)$ in the bland PVT group $(P<0.001)$.

\section{Discussion}

The importance of neoplastic and bland portal vein thrombosis discrimination in patients with $\mathrm{HCC}$ is well recognized. Bland thrombus develops from sluggish portal blood flow and can be resolved after thrombolytic and anticoagulant therapy. Neoplastic portal vein thrombus is often caused by the direct invasion of HCC and renders a patient unsuitable for aggressive treatment approaches, such as surgical resection, orthotopic liver transplantation, or chemoembolization, due to the unusually high incidence of tumor recurrence. Neoplastic PVT has also been shown to be an important factor in determining the prognosis of patients with HCC. The five-year survival after surgical resection is $12 \%-39 \%$ in patients with neoplastic vascular invasion and 59\% in those without [16-18].

Magnetic resonance imaging is of great value in the assessment of PVT [19]. To our knowledge, this is the first study of PVT analysis by SWI. We found that most SWI SI of neoplastic portal vein thrombi were similar to those of the coexisting HCC, whereas the SI of the bland thrombi were generally lower than those of the coexisting HCC. The phase value difference between neoplastic and bland PVT were statistically significant. The best cut-off value of -0.195 (in radians) gave a sensitivity of $95 \%$ and a specificity of $95.5 \%$. These results suggest that SWI is a promising tool that can be used for the diagnosis of neoplastic and bland PVT. Quantitative phase shift analysis is better than qualitative SI analysis. Neoplastic and bland thrombi are formed through different pathophysiological mechanisms. Bland thrombus develops from sluggish portal blood flow and is characterized by the presence of fibrin or blood clots without viable cells. SW imaging is an MR technology that has been shown to be sensitive to ferrihemoglobin and hemosiderin, which have only 


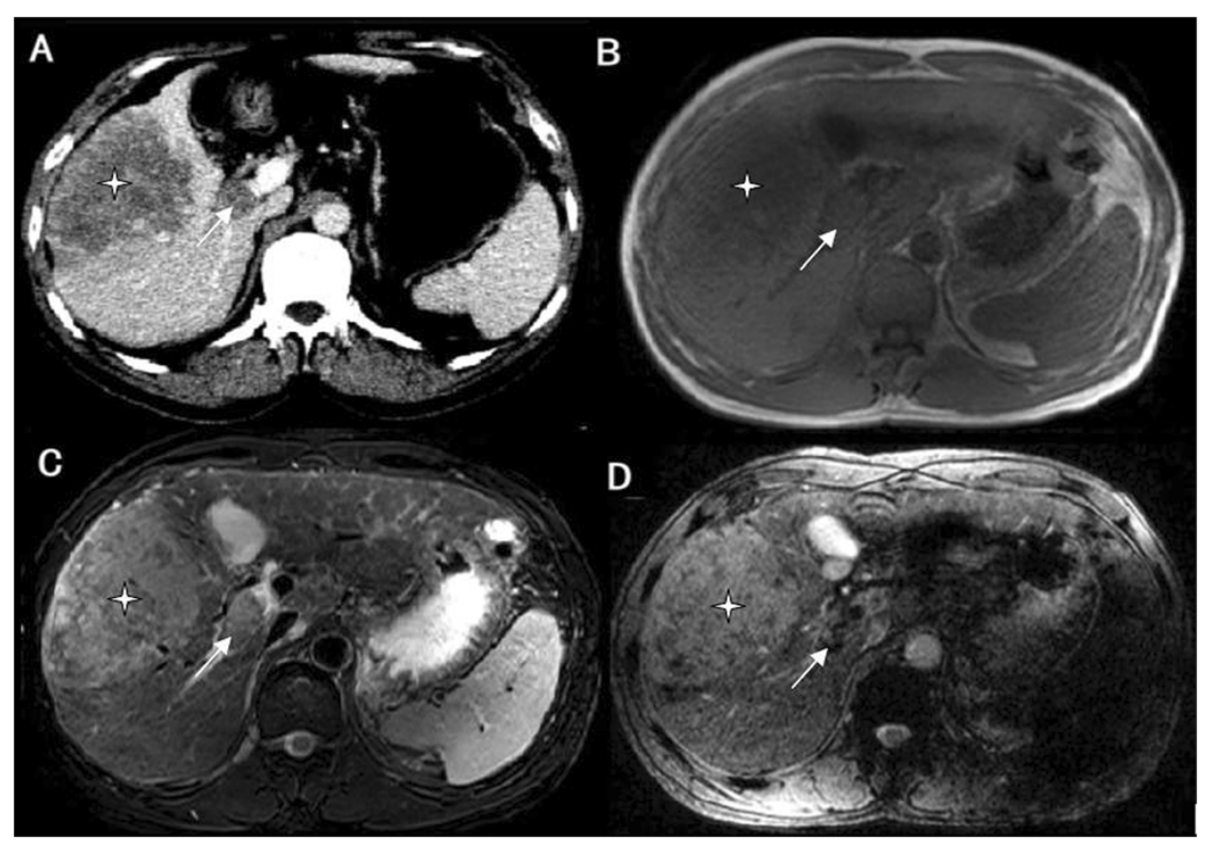

Figure 2 Bland thrombosis of the portal vein in a patient with hepatocellular carcinoma (HCC). A: Contrast-enhanced multidetector computer tomography (CE-CT); B: T1-weighted imaging (T1WI); C: T2-weighted imaging (T2WI); and D: Susceptibility-weighted imaging (SWI). HCC (*) is seen occupying the right lobe of the liver. A filling defect is noted in the right portal vein (white arrow), which exhibits lower signal intensity (SI) than the tumor by SWI.

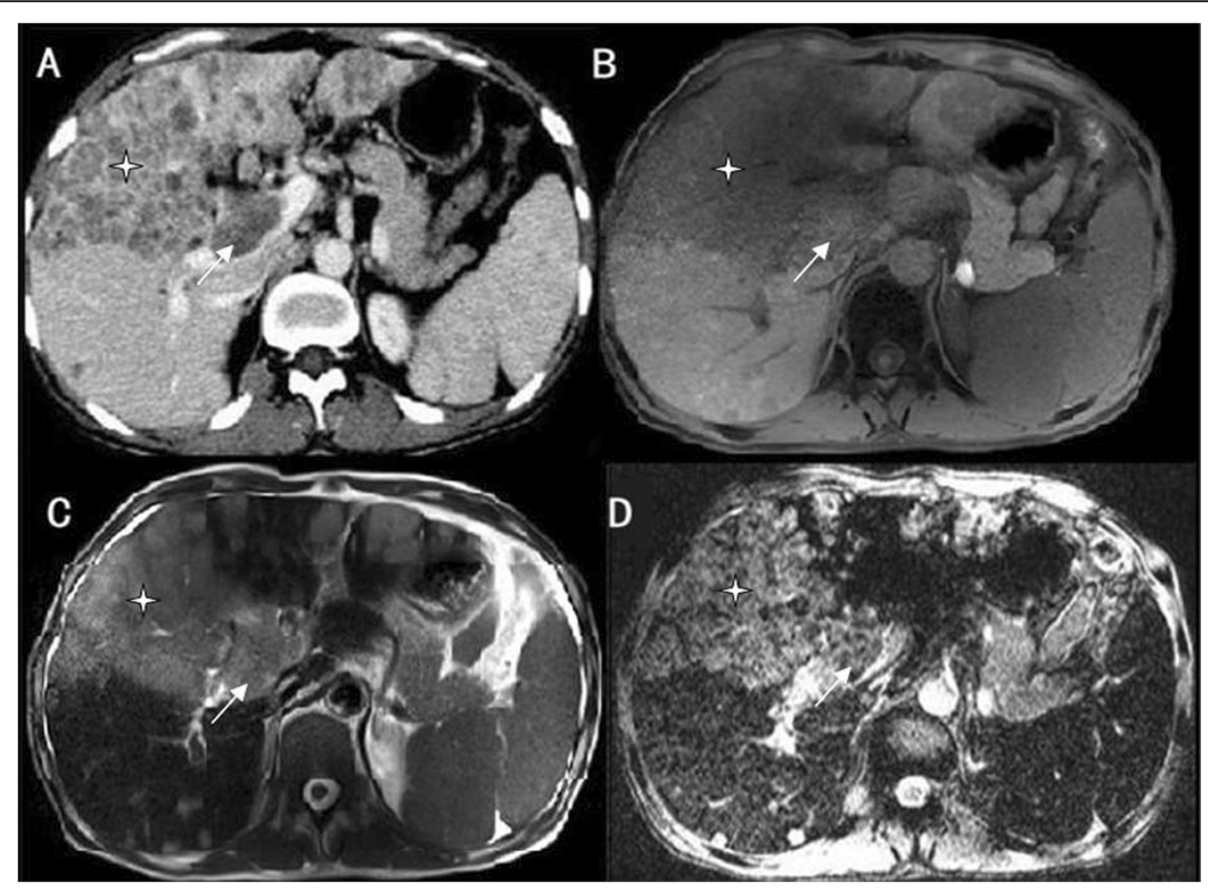

Figure 3 Neoplastic thrombosis of the portal vein in a patient with hepatocellular carcinoma (HCC). A: Contrast-enhanced multidetector computer tomography (CE-CT); B: T1-weighted imaging (T1WI); C: T2-weighted imaging (T2WI); and D: Susceptibility-weighted imaging (SWI). A large HCC $\left(^{*}\right)$ is seen in the right lobe of the liver and invades the right portal vein (white arrow). The HCC and portal vein thrombus display similar signal intensity (SI) by SW imaging. 


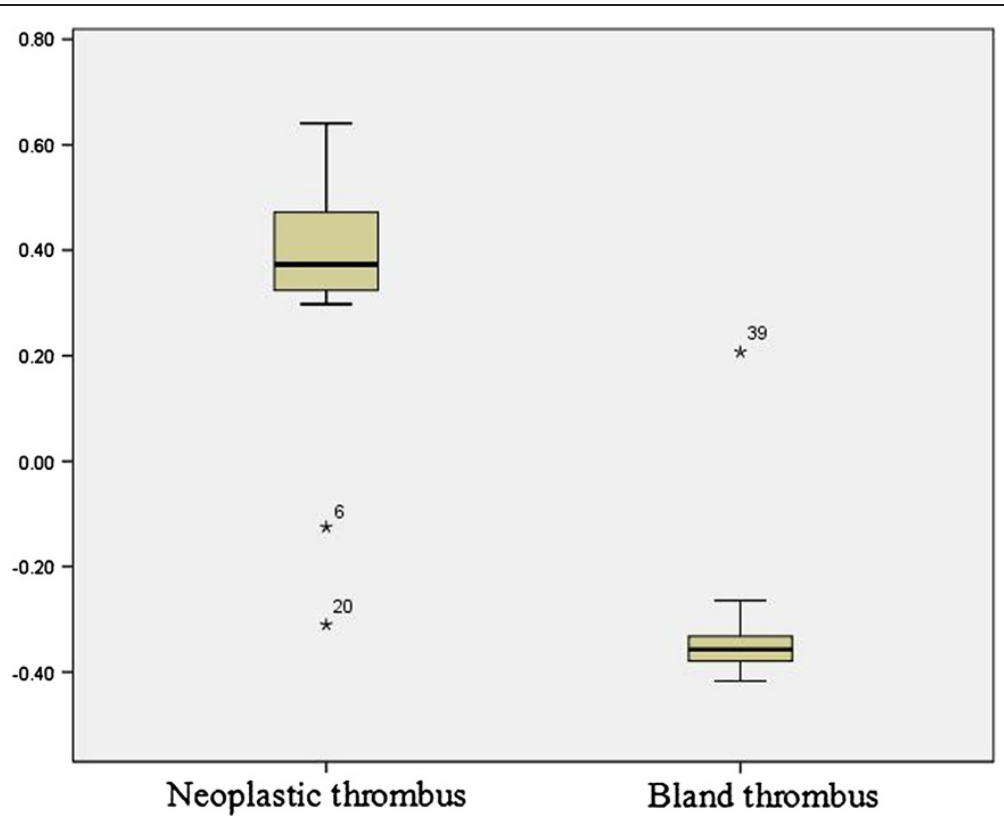

Figure 4 Phase values (in radians) of neoplastic and bland portal vein thrombi (PVT).

recently been applied in abdominal imaging. SWI does not require intravenous contrast agents or exposure to radiation. This property makes SWI suitable for repeated examinations and follow-up studies, especially for pregnant patients or for patients with a contradiction to contrast media administration.

The reference standard for characterizing portal vein thrombosis is histopathologic examination. However, portal vein thrombus biopsy is an invasive procedure with an associated risk of bleeding [20-23]. Contrast-enhanced ultrasound is notorious for being user dependent, can be difficult in obese patients and is sometimes hampered by the presence of bowel gas $[24,25]$. CE-CT is generally accepted as a reliable tool in identifying and characterizing portal vein thrombosis. The imaging criteria for malignant and benign thrombi discrimination using $\mathrm{CT}$ are well

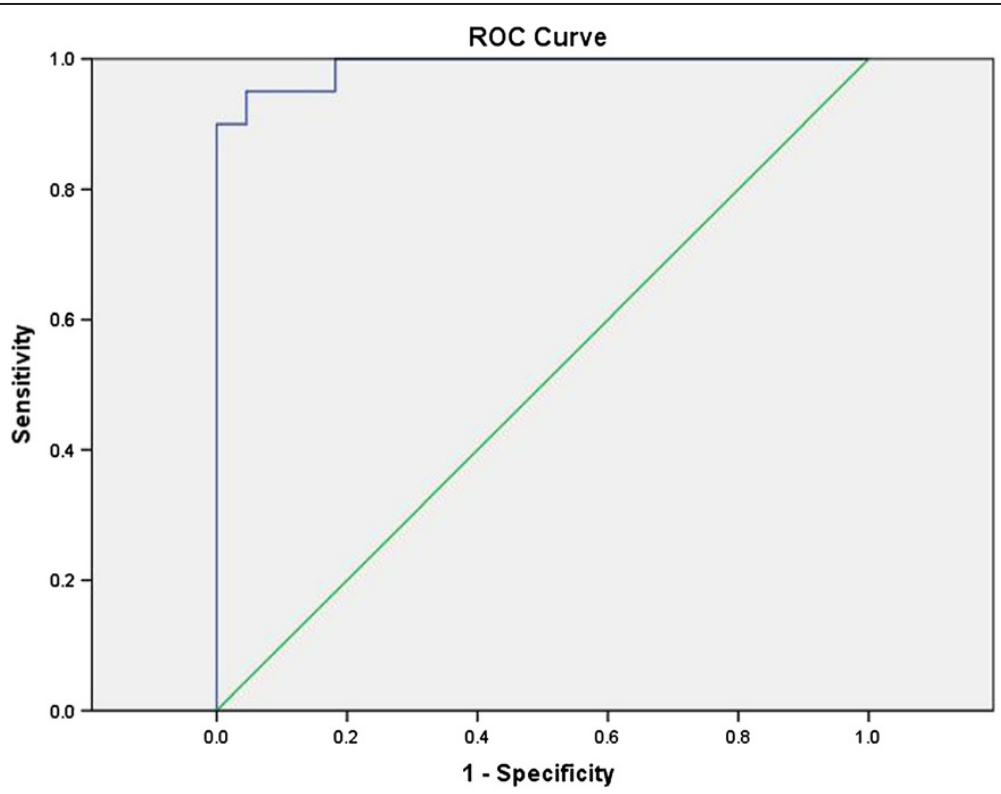

Figure 5 Receiver operating characteristics curves for phase values (in radians) in neoplastic and bland portal vein thrombi (PVT) discrimination. 
established. For example, Tublin et al. suggested that the presence of intrathrombus enhancement is highly indicative of neoplastic PVT [26]. However, CE-CT has several disadvantages, including radiation exposure and the use of contrast materials, which can lead to a fatal induction of anaphylaxis and nephropathy [27-29].

This study has several limitations. The main limitation is related to the method we used to generate the ROI for evaluation. In our study, we drew ROIs manually and calculated the phase values over the entire HCC and thrombus, which might introduce unavoidable measurement error. Secondly, due to the cross-sectional group data we could not observe the dynamic SWI in different courses of bland and neoplastic thrombi. Thirdly, because abdomen SWI is sensitive to motion artifacts from respiratory movement, our use of three consecutive breath-hold acquisitions may not be feasible in all cirrhotic patients, especially those with pulmonary compromise from hepatopulmonary syndrome or ascites. Finally, it should be noted, SI of SWI is influenced greatly by sequence parameters, especially echo time. Our results only proved the SI value under current parameters. However, phase shift value is a real, explicable index and will not change with sequence parameters. It is certainly much more reliable.

\section{Conclusions}

Neoplastic and bland PVT are both common in patients with hepatocellular carcinoma. Our results suggest that SW imaging is a promising new method for distinguishing neoplastic from bland macroscopic thrombi. The high sensitivity and specificity suggest its high value in clinical practice.

\section{Competing interests}

All authors declare that they have no competing interests.

\section{Authors' contributions}

$C L, J W$ and $X Y$ conceived and designed the experiments; $C L, J Z$ and KM performed the experiments; $C L, J H$ and $D Z$ analyzed the data. $C L$ and $X Y$ wrote the paper. All authors read and approved the final manuscript.

\section{Acknowledgement}

This study was supported by the Clinical Research Foundation of Southwest Hospital, Third Military Medical University, China (Grant No. SWH2011LC008). The sponsor of the study had no role in study design, data collection, data analysis, data interpretation, or writing of the paper.

\section{Author details}

${ }^{1}$ Department of Radiology, Southwest Hospital, Third Military Medical University, 30 Gaotanyan Road, Chongqing 400038, China. ²Department of Radiology, Wayne State University, Detroit, MI 48331, USA. ${ }^{3}$ Department of General Surgery, Southwest Hospital, Third Military Medical University, 30 Gaotanyan Road, Chongqing 400038, China.

Received: 21 May 2014 Accepted: 11 August 2014

Published: 15 August 2014

\section{References}

1. Takizawa D, Kakizaki S, Sohara N, Sato K, Takagi H, Arai H, Katakai K, Kojima A, Matsuzaki Y, Mori M: Hepatocellular carcinoma with portal vein tumor thrombosis: clinical characteristics, prognosis, and patient survival analysis. Dig Dis Sci 2007, 52:3290-3295.

2. Pirisi M, Avellini C, Fabris C, Scott C, Bardus P, Soardo G, Beltrami CA, Bartoli E: Portal vein thrombosis in hepatocellular carcinoma: age and sex distribution in an autopsy study. J Cancer Res Clin Oncol 1998, 124:397-400.

3. Sakata J, Shirai Y, Wakai T, Kaneko K, Nagahashi M, Hatakeyama K. Preoperative predictors of vascular invasion in hepatocellular carcinoma. Eur J Surg Oncol 2008, 34:900-905.

4. Ogren M, Bergqvist D, Bjorck M, Acosta S, Eriksson H, Sternby NH: Portal vein thrombosis: prevalence, patient characteristics and lifetime risk: a population study based on 23,796 consecutive autopsies. World J Gastroenterol 2006, 12:2115-2119.

5. Garcia-Pagan JCGM, Bosch J: Obstruction of the portal vein. Blackwell: Oxford, England; 2007

6. Aoki J, Iguchi Y, Kimura K, Yamashita S, Shibazaki K, Terasawa Y: Serial $\mathrm{T} 2 * \mathrm{WI}$ studies in the acute phase of cerebral venous thrombosis. Intern Med 2009, 48:383-385.

7. Haacke EM, Xu Y, Cheng YC, Reichenbach JR: Susceptibility weighted imaging (SWI). Magn Reson Med 2003, 52:612-618.

8. Haacke EM, Mittal S, Wu Z, Neelavalli J, Cheng Y: Susceptibility weighted imaging: technical aspects and clinical applications, part 1. AJNR Am J Neuroradiol 2009, 30:19-30

9. Mittal S, Wu Z, Neelavalli J, Haacke EM: Susceptibility-weighted imaging: technical aspects and clinical applications, part 2. AJNR Am J Neuroradiol 2009, 30:232-25

10. Dai Y, Zeng M, Li R, Rao S, Chen C, DelProposto Z, Haacke EM, Hu J, Renate $\mathrm{J}$ : Improving detection of siderotic nodules in cirrhotic liver with a multi-breath-hold susceptibility weighted imaging technique. J Magn Reson Imaging 2011, 34:318-325.

11. Shah ZK, McKernan MG, Hahn PF, Sahani DV: Enhancing and expansile portal vein thrombosis: value in the diagnosis of hepatocellular carcinoma in patients with multiple hepatic lesions. Am J Roentgenol 2007, 188:1320-1323.

12. Qian LJ, Zhu J, Zhuang ZG, Xia Q, Cheng YF, Li JY, Xu JR: Differentiation of neoplastic from bland macroscopic portal vein thrombi using dual-energy spectral CT imaging: a pilot study. Eur Radiol 2012, 22:2178-2185.

13. Catalano OA, Choy G, Zhu A, Hahn PF, Sahani DV: Differentiation of malignant thrombus from bland thrombus of the portal vein in patients with hepatocellular carcinoma: application of diffusion-weighted MR imaging. Radiology 2010, 254:154-162.

14. Hu S, Zhang J, Cheng C, Liu Q, Sun G, Zuo C: The role of 18F-FDGPET/CT in differentiatingmalignant from benignportal vein thrombosis. Abdom Imaging 2014. Epub ahead of print. PMID: 24913670.

15. Haacke EM, Ayaz M, Khan A, Manova ES, Krishnamurthy B, Gollapalli L, Ciulla C, Kim I, Petersen F, Kirsch W: Establishing a baseline phase behavior in magnetic resonance imaging to determine normal vs. abnormal iron content in the brain. J Magn Reson Imaging 2007, 26:256-264.

16. Minagawa M, Makuuchi M: Treatment of hepatocellular carcinoma accompanied by portal vein tumor thrombus. World J Gastroenterol 2006 12:7561-7567

17. Minagawa M, Makuuchi M, Takayama T, Ohtomo K: Selection criteria for hepatectomy in patients with hepatocellular carcinoma and portal vein tumor thrombus. Ann Surg 2001, 233:379-384.

18. Pawarode A, Voravud N, Sriuranpong V, Kullavanijaya P, Patt YZ: Natural history of untreated primary hepatocellular carcinoma: a retrospective study of 157 patients. Am J Clin Oncol 1998, 21:386-391.

19. Akin O, Dixit D, Schwartz L: Bland and tumor thrombi in abdominal malignancies: magnetic resonance imaging assessment in a large oncologic patient population. Abdom Imaging 2011, 36:62-68.

20. Kubo S, Takemura S, Yamamoto S, Hai S, Ichikawa T, Kodai S, Hiroji S, Shuto T, Hirohashi K, Tanaka H: Risk factors for massive blood loss during liver resection for hepatocellular carcinoma in patients with cirrhosis. Hepatogastroenterology 2007, 54:830-833.

21. Dusenbery D, Dodd GD, Carr BI: Percutaneous fine-needle aspiration of portal vein thrombi as a staging technique for hepatocellular carcinoma. Cytologic findings of 46 patients. Cancer 1995, 75:2057-2062. 
22. Bravo AA, Sheth SG, Chopra S: Liver biopsy. N Engl J Med 2001, 344:495-500.

23. Vilana R, Bru C, Bruix J, Castells A, Sole M, Rodes J: Fine-needle aspiration biopsy of portal vein thrombus: value in detecting malignant thrombosis. Am J Roentgenol 1993, 160:1285-1287.

24. Rossi S, Ghittoni G, Ravetta V, Torello Viera F, Rosa L, Serassi M, Scabini M, Vercelli A, Tinelli C, Dal Bello B, Burns PN, Calliada F: Contrast-enhanced ultrasonography and spiral computed tomography in the detection and characterization of portal vein thrombosis complicating hepatocellular carcinoma. Eur Radiol 2008, 18:1749-1756.

25. Tarantino L, Francica G, Sordelli I, Esposito F, Giorgio A, Sorrentino P, de Stefano G, Di Sarno A, Ferraioli G, Sperlongano P: Diagnosis of benign and malignant portal vein thrombosis in cirrhotic patients with hepatocellular carcinoma: color Doppler US, contrast-enhanced US, and fine-needle biopsy. Abdom Imaging 2006, 31:537-544.

26. Tublin ME, Dodd GD 3rd, Baron RL: Benign and malignant portal vein thrombosis: differentiation by CT characteristics. Am J Roentgenol 1997, 168:719-723.

27. Konrády A: lodine allergy-adverse reactions to contrast media. Orv Hetil 2006, 147:469-472.

28. Weisser G, Steil V, Neff KW, Büsing KA: Radiology and pregnancy Principles, general requirements and exposure. Radiology 2012, 52:81-90

29. Rajaram S, Exley CE, Fairlie F, Matthews S: Effect of antenatal iodinated contrast agent on neonatal thyroid function. Br J Radiol 2012, $85: 238-242$.

doi:10.1186/1471-2407-14-590

Cite this article as: Li et al:: Differentiation of bland from neoplastic thrombus of the portal vein in patients with hepatocellular carcinoma: application of susceptibility-weighted MR imaging. BMC Cancer 2014 14:590

\section{Submit your next manuscript to BioMed Central and take full advantage of:}

- Convenient online submission

- Thorough peer review

- No space constraints or color figure charges

- Immediate publication on acceptance

- Inclusion in PubMed, CAS, Scopus and Google Scholar

- Research which is freely available for redistribution 